\title{
Fusion Applied to Phase Altimetry in a GNSS Buoy System
}

\author{
Williams Kouassi, Georges Stienne and Serge Reboul
}

Laboratoire d'Informatique, Signal et Image de la Côte d'Opale (LISIC), Univ Lille Nord de France, F-59000 Lille, France Université du Littoral Côte d'Opale (ULCO), 50 Rue Ferdinand Buisson, BP719, 62228 Calais cedex, France

hkouassi@univ-littoral.fr 


\section{Outlines}

- Altimetry measurements

Approaches and associated geometries

Observable

Linear-Circular Regression

- Experimentations

- Synthetic data

Noise influence

Geometry influence

- Real data

Fusion application

- Conclusion 


\section{Outlines}

- Altimetry measurements

Approaches and associated geometries

Observable

Linear-Circular Regression

- Experimentations

- Synthetic data

Noise influence

Geometry influence

- Real data

Fusion application

- Conclusion 


\section{Altimetry measurement systems}

\section{Reflectometry approach}

Buoy approach
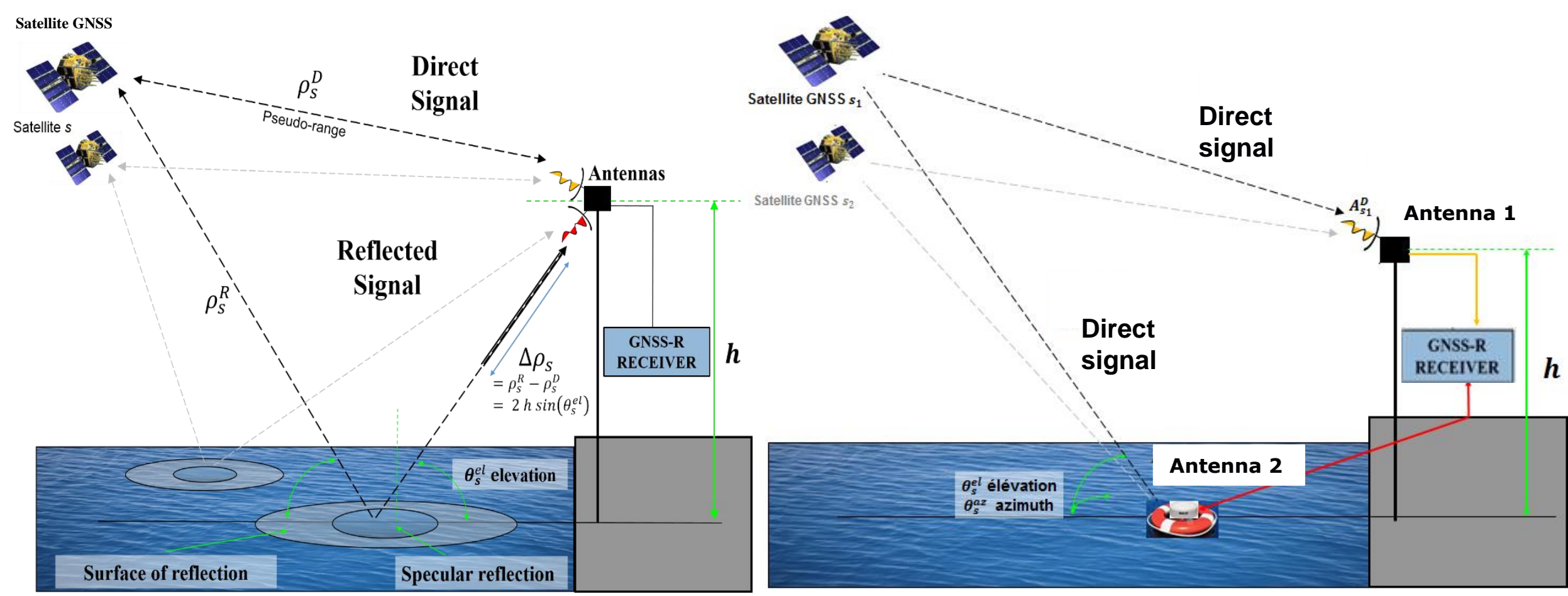


\section{Reflectometry approach's geometry}

Path difference:

Phase difference:

$$
\Delta_{s}(t)=2 h \sin \left(\theta_{s}^{e l}(t)\right)
$$

$$
\Psi_{s}(t)=\left(\frac{2 \pi}{\lambda} \Delta_{s}(t)\right) \bmod [2 \pi]
$$

$\Psi_{s}(t)=\left(\frac{4 \pi}{\lambda} h \sin \left(\theta_{s}^{e l}(t)\right)\right) \bmod [2 \pi]$

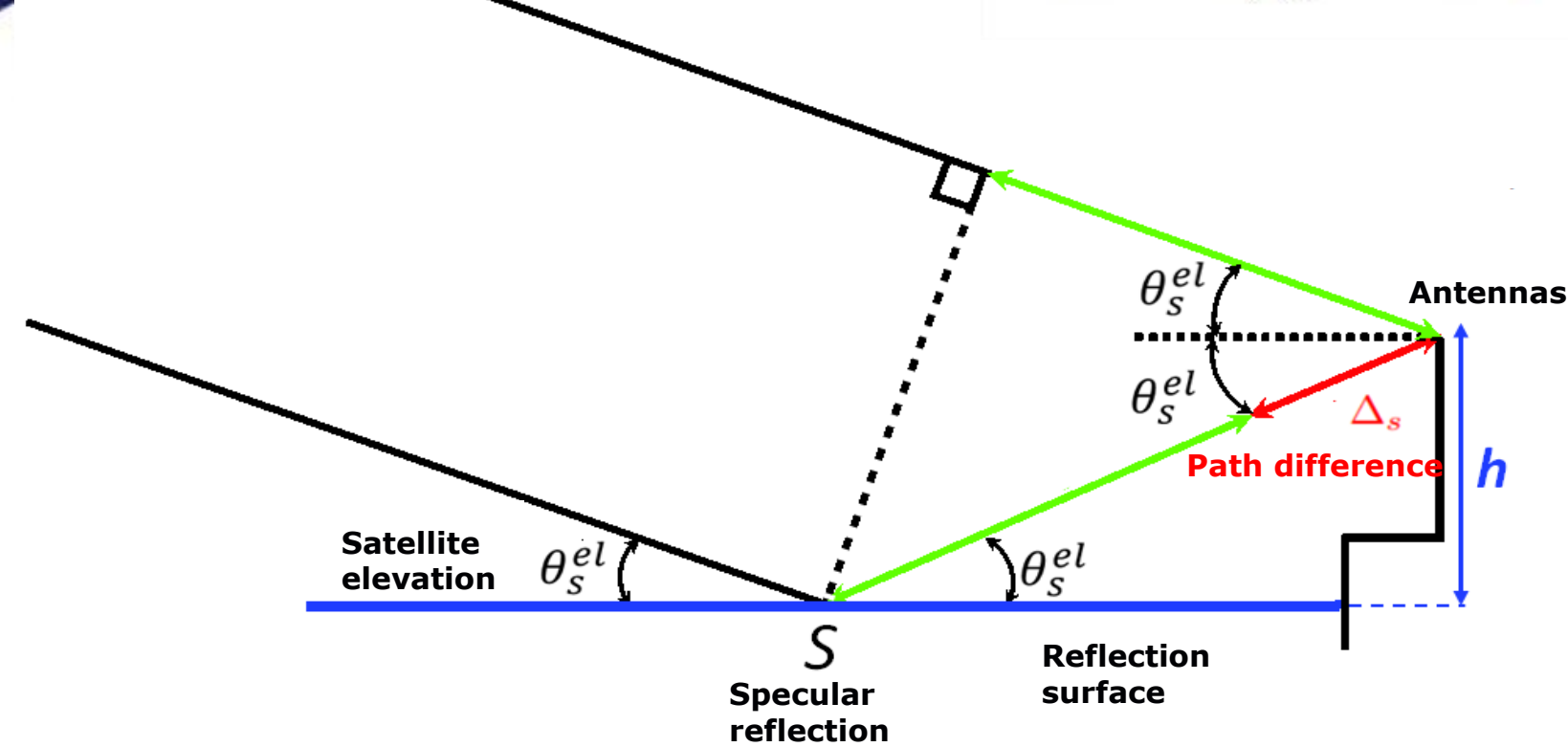

This a geometry for a reception system close to the ground. Thus, the incoming signals are supposed to be respectively parallel and we assume the reflection surface to be flat. 


\section{Buoy approach's geometry}

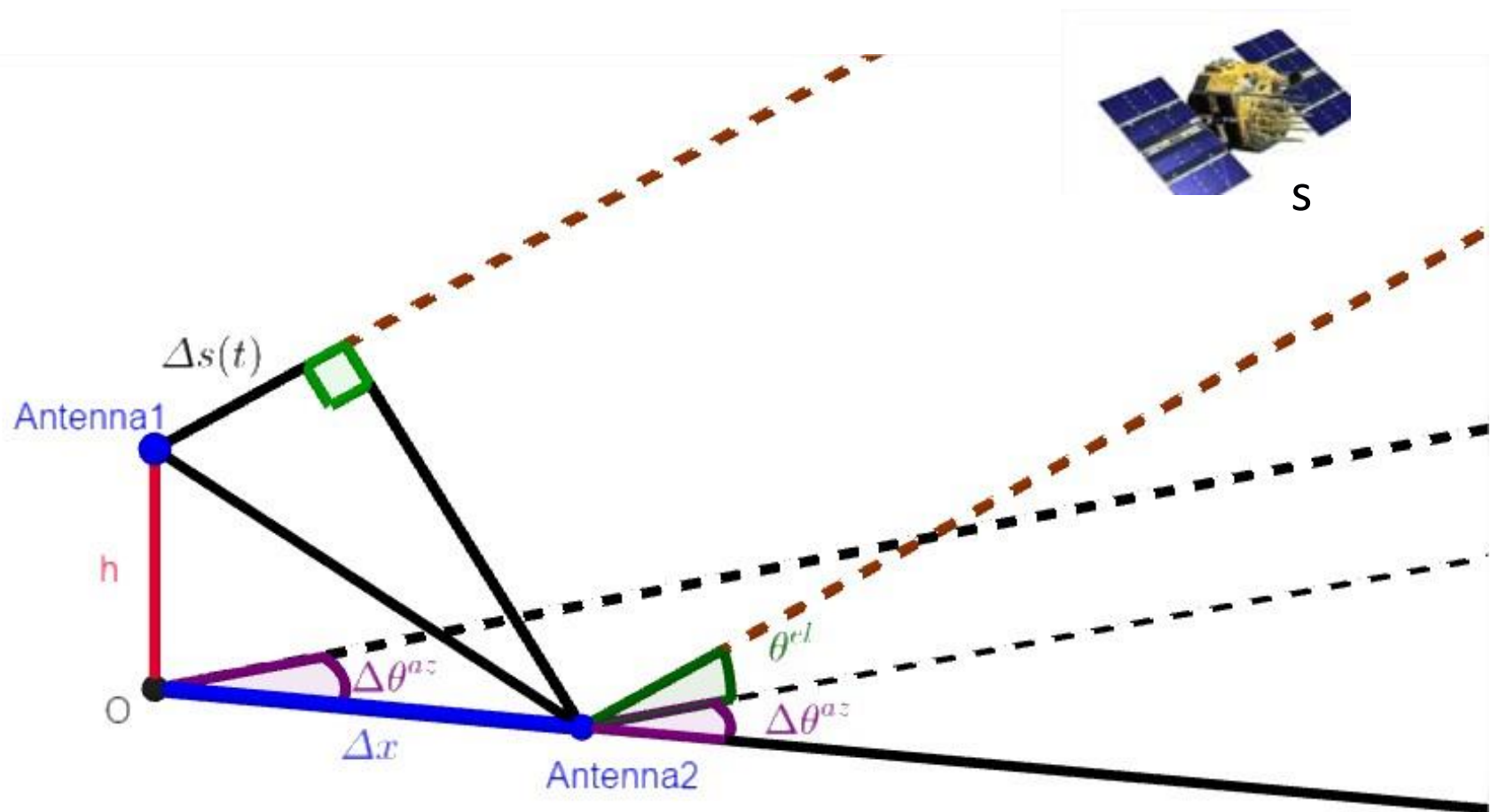

Path difference: $\quad \Delta_{s}(t)=\Delta x \cos \left(\theta_{s}^{e l}\right) \cos \left(\Delta \theta_{s}^{a z}\right)-h \sin \left(\theta_{s}^{e l}\right)$

Phase difference:

$$
\Psi_{s}(t)=\left(\frac{2 \pi \Delta_{s}(t)}{\lambda}\right) \bmod (2 \pi)
$$

$$
\Psi_{s}(t)=\left(\frac{2 \pi}{\lambda}\left(\Delta x \cos \left(\theta_{s}^{e l}\right) \cos \left(\Delta \theta_{s}^{a z}\right)-h \sin \left(\theta_{s}^{e l}\right)\right)\right) \bmod (2 \pi)
$$




\section{Observable}

Model: $\quad \Omega_{s}(t)=\left(\frac{2 \pi}{\lambda}\left(\Delta x \cos \left(\theta_{s}^{e l}\right) \cos \left(\Delta \theta_{s}^{a z}\right)-h \sin \left(\theta_{s}^{e l}\right)\right)\right)$

Measures: $\Psi_{s}(t)=\left(\frac{2 \pi}{\lambda}\left(\Delta x \cos \left(\theta_{s}^{e l}\right) \cos \left(\Delta \theta_{s}^{a z}\right)-h \sin \left(\theta_{s}^{e l}\right)\right)+\nu_{s}(t)\right) \bmod (2 \pi)$

$\nu_{s}(t)$ is a zero-mean additive noise following a Circular Normal distribution

Assuming that the path difference $\Delta x$, the elevation and azimuth angles are known using GNSS positioning and satellites ephemeris, we have:

- Known part in $[-\pi, \pi]$ :

$$
\left.\gamma(t)=\frac{2 \pi}{\lambda} \Delta x \cos \left(\Delta \theta^{a z}(t)\right) \cos \left(\theta^{e l}(t)\right)\right)
$$

$$
\gamma 1(t)=\left[\frac{2 \pi}{\lambda} \Delta x \cos \left(\Delta \theta^{a z}(t)\right) \cos \left(\theta^{e l}(t)\right)+\pi\right] \bmod (2 \pi)-\pi
$$

- Obtained model in $[-\pi, \pi]: \quad \Omega_{p i}(t)=\left[\gamma(t)-\frac{2 \pi}{\lambda} h \sin \left(\theta^{e l}(t)\right)+\pi\right] \bmod (2 \pi)-\pi$ 


\section{Observable}

- New Phase difference in $[-\pi, \pi]$ after removing $\gamma 1$ :

$$
\begin{aligned}
& \Psi_{l c r}(t)=[\Psi(t)-\gamma 1(t)+\pi] \bmod (2 \pi)-\pi \\
& \Psi_{l c r}(t)=\left[-\frac{2 \pi}{\lambda} h \sin \left(\theta^{e l}(t)\right)+\pi+\nu(t)\right] \bmod (2 \pi)-\pi
\end{aligned}
$$

$>$ This new phase observable is always a circular data depending on $h$ and the satellite elevation sine. Thus we can apply a linear-circular regression approach in order to estimate $h$. 


\section{Linear-circular regression}

$>$ We Consider $\mathrm{n}$ measurements following a linear-circular model:

$$
\Psi_{n}=\left(\alpha+\beta x_{n}+\nu_{s}(t)\right) \bmod (2 \pi)
$$

Where $X_{n}$ is a known vector and $\alpha$ and $\beta$ are the parameters to estimate.

- The Von Mises distribution of the measurements is:

$$
f\left(\Psi_{n} ; \alpha, \beta, \kappa\right)=\frac{1}{2 \pi I_{0}\left(\kappa_{s}\right)} \exp \left(\kappa \cos \left(\Psi_{n}-\left(\alpha+\beta x_{n}\right)\right)\right)
$$

- The measurements likelihood is :

$$
\begin{aligned}
L(\Psi) & =f\left(\Psi_{1} ; \alpha, \beta ; \kappa\right), \ldots, f\left(\Psi_{N} ; \alpha, \beta ; \kappa\right) \\
& =\prod_{n=1}^{N} \frac{1}{2 \pi I_{0}\left(\kappa_{s}\right)} \exp \left(\kappa \cos \left(\Psi_{n}-\left(\alpha+\beta x_{n}\right)\right)\right) \\
& =\frac{1}{\left(2 \pi I_{0}(\kappa)\right)^{N}} \exp \left(\kappa \sum_{n=1}^{N} \cos \left(\Psi_{n}-\alpha-\beta x_{n}\right)\right)
\end{aligned}
$$




\section{Linear-circular regression}

- The log-likelihood:

$\ln L(\Psi)=-N \ln \left(\left(2 \pi I_{0}(\kappa)\right)\right)+\kappa \sum_{n=1}^{N} \cos \left(\Psi_{n}-\alpha-\beta x_{n}\right)$

- Maximizing the log-likelihood, we derive an estimation of $\alpha$ :

$$
\frac{\partial L(\Psi)}{\partial \alpha}=0 \quad \longrightarrow \quad \hat{\alpha}=\arctan ^{*}\left(\frac{\sum_{n=1}^{N} \sin \left(\Psi_{n}-\beta x_{n}\right)}{\sum_{n=1}^{N} \cos \left(\Psi_{n}-\beta x_{n}\right)}\right)
$$

- The maximum likelihood estimates of $\alpha$ and $\beta$ can be obtained recursively using the Newton-Raphson Algorithm:

$$
\hat{\alpha}^{i}=\arctan ^{*}\left(\frac{\sum_{n=1}^{N} \sin \left(\Psi_{n}-\hat{\beta}^{i} x_{n}\right)}{\sum_{n=1}^{N} \cos \left(\Psi_{n}-\hat{\beta}^{i} x_{n}\right)}\right)
$$

$\hat{\beta}^{i+1}=\hat{\beta}^{i}+g\left(\frac{\partial^{2} L(\Psi)}{\partial \beta^{2}}\right)^{-1}\left(\frac{\partial L(\Psi)}{\partial \beta}\right) \quad \longrightarrow \quad \hat{\beta}^{i+1}=\hat{\beta}^{i}+g\left(\frac{\sum_{n=1}^{N} x_{n} \sin \left(\Psi-\left(\hat{\alpha}^{i}+\hat{\beta}^{i} x_{n}\right)\right)}{\sum_{n=1}^{N}\left(x_{n}\right)^{2} \cos \left(\Psi-\left(\hat{\alpha}^{i}+\hat{\beta}^{i} x_{n}\right)\right)}\right)$ 


\section{Linear-circular regression}

In our application:

$$
\Psi_{n}=\left(\alpha+\beta x_{n}+\nu_{s}(t)\right) \bmod (2 \pi) \quad \text { With : }
$$

- $x_{n}=\sin \theta_{n}^{e l}, n \in 1 . . N$

- $\alpha=0$

- $\hat{\beta}=\frac{-2 \pi}{\lambda} \hat{h}$
Where $\mathrm{n}$ is the sampling instant

Obtained directly from the expression of $\Psi_{l c r}$

Thus $\hat{h}$ can be estimated from the expression of $\widehat{\beta}$ 


\section{Outlines}

- Altimetry measurements

Approaches and associated geometries

Observable

Linear-Circular Regression

- Experimentations

- Synthetic data

Noise influence

Geometry influence

- Real data

Fusion application

- Conclusion 


\section{Evaluations on synthetic data}

\section{Noise level influence:}

- Simulations parameters:

- $\Delta \mathrm{x}=20 \mathrm{~m}$

- Height to estimate: $h=20 \mathrm{~m}$

- Number of realizations: 100

- Carrier frequency: $1,57542 \mathrm{GHz}$

- 3s of signal

- Mean of elevation: $30^{\circ}$

- Mean of $\partial \theta(t) / \partial t: 0,005^{\circ} / \mathrm{s}$

\begin{tabular}{|c|c|c|c|c|}
\hline$C / N_{0}(\mathrm{~dB} . \mathrm{Hz})$ & 50 & 40 & 30 & 20 \\
\hline RMSE $(\mathrm{cm})$ & 0,0402 & 0,0675 & 0,1884 & 0,2832 \\
\hline
\end{tabular}

- Conclusion: We notice that the accuracy obtained on the estimated height depends on the noise level. The lower the noise is the better is the precision of the estimator. However, the Linear-Circular regression method is robust to higher noise levels 
Evaluations on synthetic data

\section{Geometry Influence (C/NO = 30dB.Hz):}

$30^{\circ}$ average elevation sine

\begin{tabular}{|c|c|c|c|}
\hline$\frac{\partial\left(\theta^{e l}(t)\right)}{\partial(t)}\left({ }^{\circ} / \mathrm{s}\right)$ & $8.10^{-3}$ & $5.10^{-3}$ & $2.10^{-3}$ \\
\hline$\frac{\partial\left(\sin \theta^{e l}(t)\right)}{\partial(t)}\left(\mathrm{s}^{-1}\right)$ & $1,0300.10^{-4}$ & $6,9033.10^{-5}$ & $2,9261.10^{-5}$ \\
\hline Estimated RMSE $(\mathrm{cm})$ & 0,1140 & 0,1884 & 0,4053 \\
\hline
\end{tabular}

$70^{\circ}$ average elevation sine

\begin{tabular}{|c|c|c|c|}
\hline$\frac{\partial\left(\theta^{e l}(t)\right)}{\partial(t)}(\circ / \mathrm{s})$ & $8.10^{-3}$ & $5.10^{-3}$ & $2.10^{-3}$ \\
\hline$\frac{\partial\left(\sin \theta^{e l}(t)\right)}{\partial(t)}\left(\mathrm{s}^{-1}\right)$ & $1,9281.10^{-5}$ & $1,8830.10^{-5}$ & $1,0200.10^{-5}$ \\
\hline Estimated RMSE $(\mathrm{cm})$ & 0,6089 & 0,6859 & 1,2727 \\
\hline
\end{tabular}

Conclusion: For a visible satellite, the variation of its elevation sine over the time must be fast for a better precision on the estimate. The best satellites in this case are those for which the elevation sine is varies in an interval which is on the increasing part of the representative sinus curve. For satellites whose elevation sine varies over a given interval which is close to the top of the representative sinus curve, their elevation sine varies slowly and the accuracy is worst. 


\section{Fusion Application: Real data}

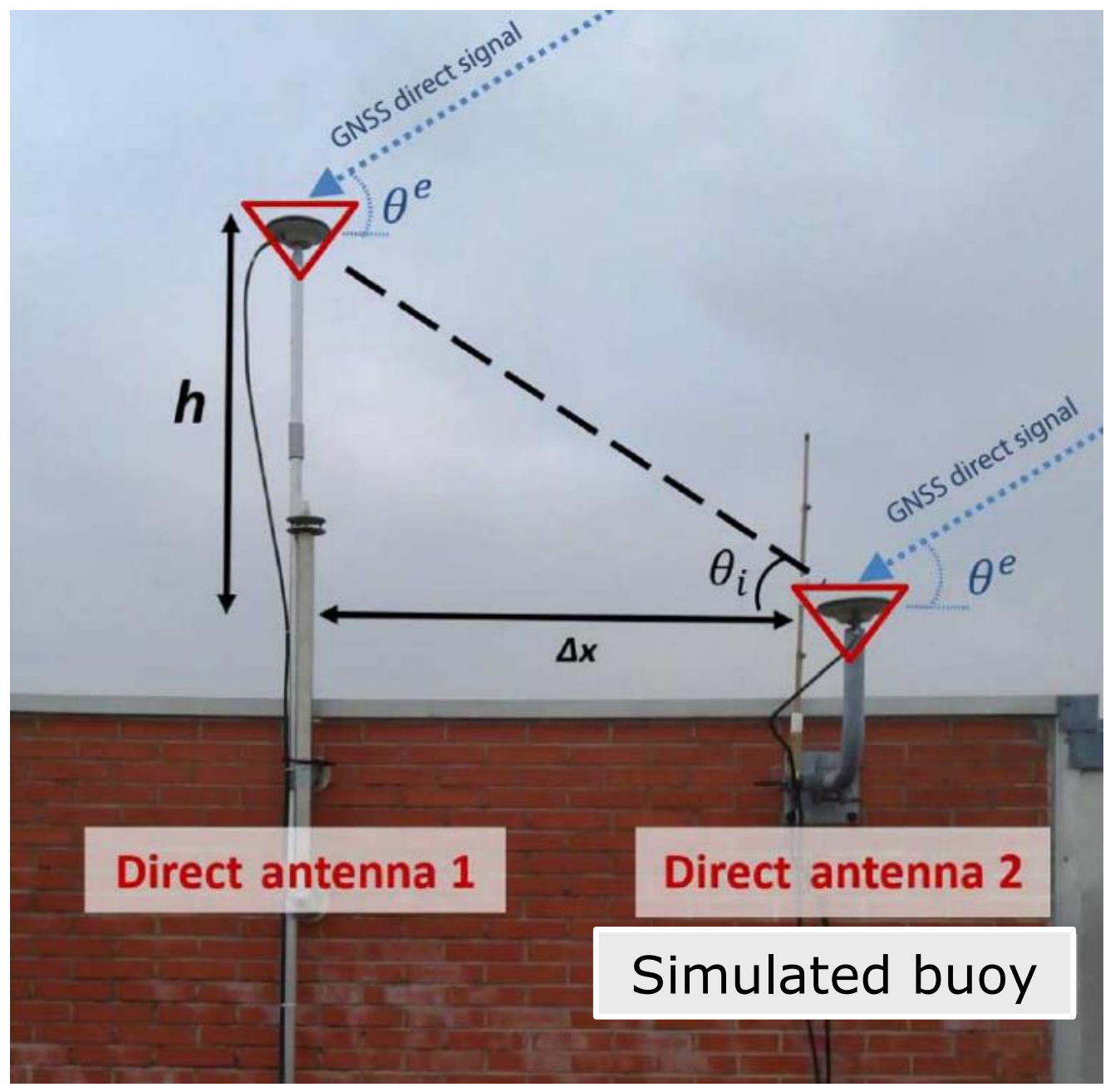

In our implementation on real data, we use an experimental setup that allows recording of two direct signals. This setup is presented in this figure.

In the proposed setup, the GNSS signal are received using two GPS antennas. The processing are done on a software receiver. For this experimentation, we record $18 \mathrm{~s}$ of data for a distance and a height between the two antennas of $\Delta x=144 \mathrm{~cm}$ and $\mathrm{h}=51,30 \mathrm{~cm}$. 


\section{Experimentations}

\section{Fusion Application: Real data}

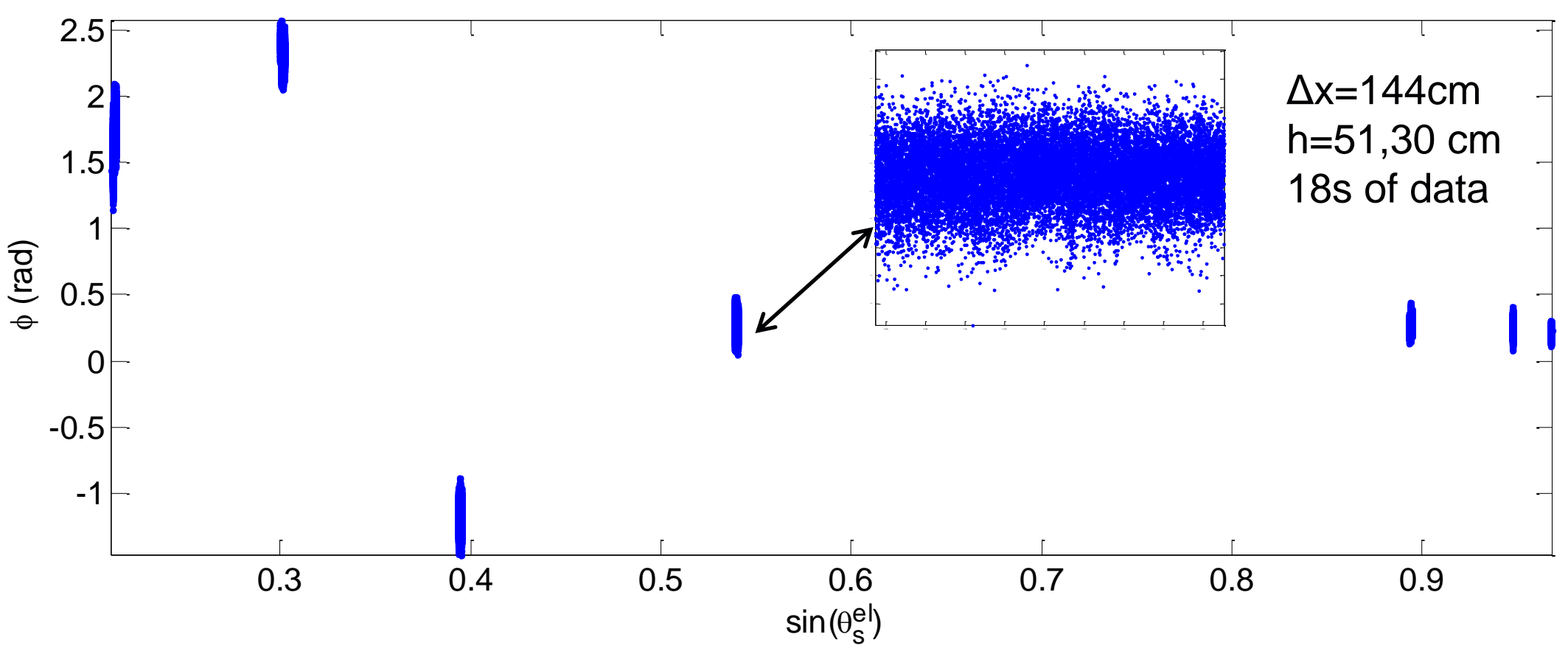

In the experimentation using synthetic data, we considered $\mathrm{N}$ phase measurements. In the case of the fusion, the amount of data is extended to $\mathrm{N}^{*} \mathrm{~S}$ where $\mathrm{S}$ is the number of satellites. Thus, the advantage here, is to increase the amount of data and the elevation sine span like in this figure. Then, we show (in the next table) that we can reach a centimeter precision using this technic. 


\section{Experimentations}

\section{Fusion Application: Real data}

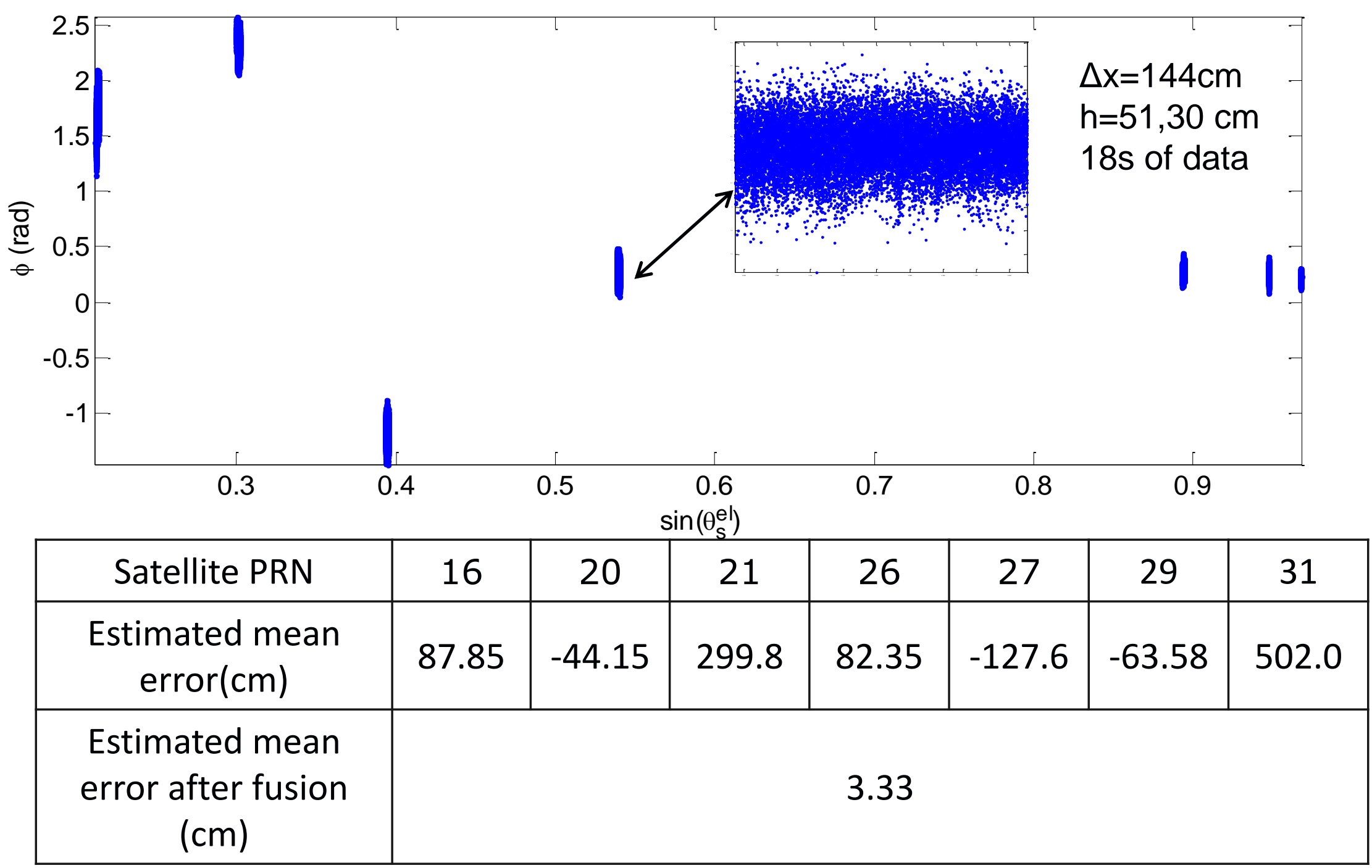




\section{Conclusion}

- measures in $z$ axis in GPS are less accurate than $x$ and $y$

- Use of altimetry for height measurements

- Linear-Circular Regression: Robust to high noise levels

- High influence of the elevation sine on the accuracy

- Centimeter precision

- Better results using data fusion over several satellites

Perspectives:

- Real setting with a buoy

- Joint estimation of $\Delta x$ and $\mathrm{h}$

The autors would like to acknowledge the financial support from the CPER MARCO program 